\title{
Dipole Moment Studies. V.* The Dipole Moments of the Methylphosphine Boranes $\dagger$
}

\author{
JOSEPH G. MORSE \\ Department of Chemistry, University of Michigan, Ann Arbor, Michigan 48104
}

(Received 21 April 1972)

\begin{abstract}
Dipole moments of methylphosphine borane, dimethylphosphine borane, and trimethylphosphine borane were determined in benzene solution. After correction for solvent effects the values obtained were: $\mathrm{CH}_{3} \mathrm{PH}_{2} \mathrm{BH}_{3}, 4.58 \mathrm{D} ;\left(\mathrm{CH}_{3}\right)_{2} \mathrm{PHBH}_{3}, 4.78 \mathrm{D} ;\left(\mathrm{CH}_{3}\right)_{3} \mathrm{PBH}_{3}, 4.97 \mathrm{D}$. The variation in these moments is interpreted in terms of a polarization model.
\end{abstract}

An earlier paper in this series described the determination of the dipole moments of the methylamine boranes. ${ }^{1}$ A polarization model was designed to interpret trends in dipole moments for alkylamines, the alkylphosphines, and the amine boranes (see asterisk footnote). This model quite clearly dictated the trend to be expected in a series of alkylphosphine boranes. According to the model, substitution of methyl groups for the protons of phosphine should lead to an increase in the polarizability of the lone pair of electrons on phosphorus. It is expected that the donation of electrons to the boron atom in the phosphine boranes (bond length of $\sim 1.9 \AA$ ) should lead to a dipole moment large enough to dominate the observed molecular moment. The more easily the electron pair is distorted, the larger this moment

FIG. 1. Dipole moments of methylamines, phosphines, and their borane adducts.

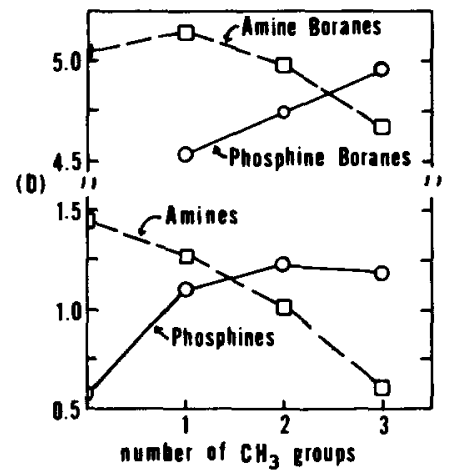

should be. Thus one should observe a stepwise increase in the dipole moments of the methylphosphine boranes at each substitution of methyl for proton. It was the intent of this study to determine the reliability of this prediction.

Since the low vapor pressures of the phosphine boranes precludes determination of their dipole moments in the vapor phase by the heterodyne beat method, the determinations were made in benzene solution. Data on phosphine borane, $\mathrm{PH}_{3} \mathrm{BH}_{3}$, are not yet available.

\section{EXPERIMENTAL}

\section{Materials Used}

The methylphosphine boranes were prepared as described in the literature ${ }^{2}$ from diborane and the appropriate methyl substituted phosphine.
Solvent benzene was reagent grade and thiophene free (Allied Chem. C.). It was dried over $\mathrm{NaH}$ then distilled in the vacuum line.

\section{Preparation of Solutions}

Solutions were prepared under vacuum then brought to atmospheric pressure by the addition of dry $\mathrm{N}_{2}$ gas. Solutions were maintained under a dry nitrogen atmosphere throughout the measurements. Details of the procedure are given elsewhere (see dagger footnote).

\section{Measurements on Solutions}

(a) Dielectric constants were determined using apparatus previously described. ${ }^{3}$ Solutions were added and removed from the cell by hypodermic syringe. Pure,

FIG. 2. Dipole moments of methylamine boranes corrected for the lone-pair moments of the free amines and dipole moments of the methylphosphine boranes.

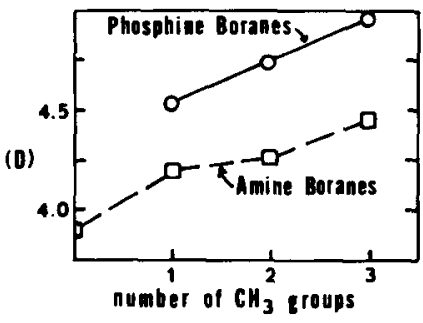

dry benzene was used as a standard with its dielectric constant taken as 2.2725 . $4 \mathrm{a}$

(b) Density measurements were made using a thermostated pycnometer $\left(25.0^{\circ} \pm 0.02\right)$ of about $15 \mathrm{ml}$ capacity. The exact volume was determined by measuring the height of the liquid above or below a fixed reference mark on the capillary neck with a cathetometer and comparing this to a previously prepared calibration chart. The density of pure benzene was taken as $0.87378 \mathrm{~g} / \mathrm{cc}^{4 \mathrm{~b}}$

(c) Refractive index measurements were made with a high precision Abbe refractometer at the sodium D line.

\section{RESULTS}

Experimental data are summarized in Table I. The dipole moments of the methylphosphine boranes were were calculated from the data by two different methods. A "conventional" value was obtained using the Hedestrand procedure ${ }^{40}\left(\mu_{\text {conv }}\right) . \beta$ and $\gamma$, the coefficients of 
TABLE I. Data for calculation of dipole moments of methylphosphine boranes.

\begin{tabular}{lccccccc}
\hline & $\alpha$ & $\beta$ & $\gamma$ & $P$ & $R$ & $\mu_{\text {conv }}$ & $\mu_{\text {ell }}$ \\
\hline $\mathrm{CH}_{3} \mathrm{PH}_{2} \mathrm{BH}_{3}$ & $25.67 \pm 0.13$ & $-0.0716 \pm 0.0055$ & $-0.0556 \pm 0.0042$ & 400.6 & 22.07 & $4.29 \pm 0.01$ & 4.58 \\
$\left(\mathrm{CH}_{3}\right)_{2} \mathrm{PHBH}_{3}$ & $29.72 \pm 0.18$ & $-0.109 \pm 0.008$ & $-0.0798 \pm 0.0038$ & 465.0 & 26.22 & $4.62 \pm 0.01$ & 4.78 \\
$\left(\mathrm{CH}_{3}\right)_{3} \mathrm{PBH}_{3}$ & $33.53 \pm 0.10$ & $-0.1476 \pm 0.0071$ & $-0.1745 \pm 0.0048$ & 527.8 & 31.97 & $4.91 \pm 0.01$ & 4.97 \\
\hline \hline
\end{tabular}

change in density and refractive index with mole fraction of solute, $x$, were obtained by the method of least squares. The corresponding coefficient of change in dielectric constant, $\alpha$ was calculated by

$$
\alpha=\left(\sum \epsilon-n \epsilon_{1}\right) / \sum x,
$$

where $n$ is the number of measurements and the subscript, one, indicates pure solvent. This method was used for $\alpha$ because the $\epsilon$ values were calculated relative to $\epsilon_{1}$ so that $\epsilon=\epsilon_{1}$ at $x=0$ is a fixed point on the line. Within the limits of random error there was no deviation from linearity in any of these plots. All concentrations were in the range $0.001-0.025$ mole fraction with most values below 0.015 . Error limits given are standard error. Hedestrand's equation for molar polarization may be written for benzene solutions at $25^{\circ} \mathrm{C}$ as

$$
P=0.34086 M+14.692 \alpha-30.472 \beta .
$$

Molar refraction, $R$, was obtained using

$$
R=0.33557 M-30.000 \beta+14.886 \gamma,
$$

where $M$ is the molecular weight of solute. Atomic polarization was approximated as $0.10 R$. The dipole moments were then obtained as

$$
\mu_{\text {oonv }}=0.2212(P-1.10 R)^{1 / 2} \text {. }
$$

The $\mu_{\text {conv }}$ values were corrected for solvent effects according to the method described in Ref. 5. The equations used are given elsewhere. (See Refs. 5 and 6 and dagger footnote.) The cavity shapes were estimated using mean value of bond lengths in similar compounds and assuming tetrahedral geometry. The elliptical parameters used in the calculations were biased toward spherical when there was question concerning cavity shape. The resulting values are given as $\mu_{\mathrm{ell}}$. This value is assumed to be most nearly correct for subsequent discussion [Note added in proof: This conclusion is supported by a microwave study ${ }^{\mathrm{Ba}}$ of two of these compounds reported after completion of this manuscript.], but it can be seen in Table $I$ that the same trend in moments would result if the other values were used. There was no evidence for association in benzene solution as was observed with the amineboranes.

\section{DISCUSSION}

The results are plotted in Fig. 1 as a function of methyl substitution. Dipole moment data for the methylamines, ${ }^{7,8 a, 9}$ their borane adducts, ${ }^{1}$ and the methylphosphines ${ }^{8 b, 10,11}$ are also included. The striking contrast between the trends in the amines and phosphines as been attributed, though not very satisfactorily in our judgment, to variations in hybridization. ${ }^{12}$ The polarization model of this laboratory (see asterisk footnote) attributes the dominant role in the variation of amine moments to the contribution of the lone pair of electrons. This is thought to decrease with increasing alkyl substitution due to loosening of the lone pair and its resulting relaxation toward the relatively positive carbon atoms. In the phosphines, with a negligible lone pair contribution to the net moment, the trend is more nearly what conventional inductive effect ideas would suggest.

The methylamine boranes provided an excellent illustration of these ideas. In Ref. 1 a plot of the methylamine borane dipole moments was made after data were corrected to eliminate the lone pair contribution. This curve should represent very nearly the shape of the curve for the methylphosphine boranes if the lone pair moment is not, in fact, a major contributor to the dipole moments of the methylphosphines. The values for the amine-boranes corrected in this fashion are shown with the phosphine borane results in Fig. 2. The trend observed for the methylphosphine boranes is, indeed, very similar to that for the "corrected" amine boranes.

\section{ACKNOWLEDGMENTS}

The authors thank Dr. K. W. Morse for samples of methylphosphines. A National Science Foundation Cooperative Fellowship for J.G.M. is gratefully acknowledged.

* Paper IV: J. R. Weaver and R. W. Parry, Inorg. Chem. 5, 718 (1966)

† Abstracted from the Ph.D. thesis of J. G. Morse, The University of Michigan, 1967.

I Present address: Department of Chemistry, Utah State University, Logan, Utah.

$\$$ Present address: Department of Chemistry, University of Utah, Salt Lake City, Utah 84112.

I J. R. Weaver and R. W. Parry, Inorg. Chem. 5, 713 (1966).

2 A. B. Burg and R. I. Wagner, J. Am. Chem. Soc., 75, 3872 (1953)

${ }_{3}^{3}$ J. R. Weaver, S. G. Shore, and R. W. Parry, J. Chem. Phys. 29, 1 (1958).

(a) R. J. W. LeFevre, Dipole Moments (Methuen, London, 1958), (a) p. 2, (b) p. 46, (c) p. 55.

5 J. R. Weaver and R. W. Parry, Inorg. Chem. 5, 703 (1966).

- G. Kodama, J. R. Weaver, J. LaRochelle, and R. W. Parry Inorg. Chem. 5, 710 (1966). 
6a P. S. Bryan and Robert L. Kuczkowski, Inorg. Chem. 11, 553 (1972), report 4.99 $\pm 0.2 \mathrm{D}$ for $\left(\mathrm{CH}_{8}\right)_{3} \mathrm{PBH}_{3}$ and $4.66 \pm 0.05 \mathrm{D}$ for $\mathrm{CH}_{3} \mathrm{PH}_{2} \mathrm{BH}_{3}$.

${ }^{7}$ D. R. Lide, Jr., J. Chem. Phys. 27, 343 (1957); 20, 1812 (1952).

${ }^{8}$ (a) D. R. Lide, Jr., and D. E. Mann, J. Chem. Phys. (a) 28, 572 (1958). (b) 29,914 (1958).
${ }^{9}$ R. J. W. LeFevre and P. Russell, Trans. Faraday Soc. 43, 374 (1947).

${ }^{10}$ T. Kojima, E. Breig, and C. C. Lin, J. Chem. Phys. 35, 2139 (1961).

${ }_{11}$ R. Nelson, J. Chem. Phys. 39, 2382 (1963).

12 R. F. Hudson, Siructure and Mechanism in Organophosphorus Chemistry (Academic, New York, 1965).

\title{
Dipole Moment Studies. VI. Dipole Moments of the Propylphosphines
}

\author{
Joseph G. Morse and R. W. Parry* \\ Department of Chemistry, The University of Michigan, Ann Arbor, Michigan and Department of Chemistry, \\ Utah State University, Logan, Utah 84321
}

(Received 21 April 1972)

\begin{abstract}
The dipole moments of mono-n-propylphosphine and mono- $i$-propylphosphine, as measured in the gas phase with a heterodyne beat apparatus, are $1.09 \pm 0.06$ and $1.22 \pm 0.03 \mathrm{D}$, respectively. The moments of mono- $n$-propylphosphine, di- $n$-propylphosphine, and tri- $n$-propylphosphine as measured in benzene solution and corrected for solvent effect are $1.17 \pm 0.05,1.22 \pm 0.05$, and $1.16 \pm 0.08 \mathrm{D}$, respectively. The moments for mono-i-propylphosphine, di-i-propylphosphine, and tri-i-propylphosphine as measured in benzene solution and corrected for solvent effects are $1.13 \pm 0.03,1.32 \pm 0.12$, and $1.32 \pm 0.04 \mathrm{D}$, respectively. Discrepancies between gas phase and solution values are considered, and values for each series are discussed in terms of a bond polarization model.
\end{abstract}

Earlier papers in this series ${ }^{1-4}$ reported values for the dipole moments of the ethylphosphines and the methylamine boranes. A polarization-bond moment-lone-pair moment model was used to interpret the data and to explain striking contrasts in the trends for the dipole moments of the alkylamines and alkylphosphines. ${ }^{4}$ The present paper provides dipole moment data on the $n$-propyl- and the $i$-propylphosphines thus permitting an extension of the model.

The literature ${ }^{5}$ contains the value $1.48 \mathrm{D}$ as the moment for tri- $n$-propylphosphine, but no values for other members of the series. Because of problems in measuring precise moments for all members of this series by any available technique, the entire series has been reexamined by a single procedure using methods which have been effectively applied to the lighter members of the phosphine series. As the data clearly show, some uncertainty remains in the measurements of the propylphosphines but trends are clearly apparent.

The experimental moments of the mono- $n$-propylphosphine and the mono-i-propylphosphine have been measured in the vapor state. Comparable measurements on the monopropylphosphines in solution provide an evaluation of available solution correction procedures. ${ }^{1}$ Measurements on both of the di- and on both of the tripropylphosphines had to be made in benzene solution because of low sample volatility. No vapor phase comparison was possible on these four molecules. Because of the complexity of the propylphosphines, precise microwave dipole values are not yet available for any member of the series. The microwave problem is definitely not trivial and has not yet been solved.

\section{RESULTS}

\section{Gas Phase Measurements on Primary Propylphosphines}

Gas phase data were reduced by plotting molar polarization of the gas against the inverse of the temperature (Debye method). Details are given in the experimental section. The rather large standard error limits given for the gas phase measurements, particularly for $n-\mathrm{C}_{3} \mathrm{H}_{7} \mathrm{PH}_{2}$, are a result of the low volatility of the phosphine and of some adsorption of the vapor in the vacuum system.

\section{Solution Measurements on Propylphosphines}

Molecular dipole moments were calculated using the standard expression $\mu=0.2212\left(P_{0}\right)^{1 / 2}$, where $P_{0}$ is the orientation polarization; this was obtained as described in the experimental section. The resulting values, which include no correction for solvent effect, are referred to as conventional moments and are listed in Table $I$ as $\mu_{\text {conv }}$. Standard error limits represent precision, not accuracy. These conventional values were corrected for solvent effects using two procedures: (1) the spherical cavity model of Onsager ${ }^{6}$ and (2) the elliptical cavity model of Ref. 1 . Known values for the dielectric constant of the solvent and measured values for the distortion polarization of both solvent and solute were used. The shape of the elliptical cavity used in Method 2 is important since it compensates in part for moments induced in the solvent by the polar solute. Parameters for the cavity were taken from bond angles, bond 